\title{
Sharp bounds for a ratio of the $q$-gamma function in terms of the $q$-digamma function
}

\section{Faris Alzahrani ${ }^{1}$, Ahmed Salem ${ }^{1 *}$ (D) and Moustafa El-Shahed ${ }^{2}$}

\section{"Correspondence:}

ahmedsalem74@hotmail.com

'Department of Mathematics,

Faculty of Science, King Abdulaziz

University, P.O. Box 80203, Jeddah,

21589, Saudi Arabia

Full list of author information is

available at the end of the article

\begin{abstract}
In the present paper, we introduce sharp upper and lower bounds to the ratio of two $q$-gamma functions $\Gamma_{q}(x+1) / \Gamma_{q}(x+s)$ for all real number $s$ and $0<q \neq 1$ in terms of the $q$-digamma function. Our results refine the results of Ismail and Muldoon (Internat. Ser. Numer. Math., vol. 119, pp. 309-323, 1994) and give the answer to the open problem posed by Alzer (Math. Nachr. 222(1):5-14, 2001). Also, for the classical gamma function, our results give a Kershaw inequality for all $0<s<1$ when letting $q \rightarrow 1$ and a new inequality for all $s>1$.
\end{abstract}

MSC: 33D05; 26D07; 26A48

Keywords: $q$-Gamma function; $q$-Digamma function; Inequalities; Completely monotonic function

\section{Introduction}

There exist several published articles providing different upper and lower bounds for the ratio of two gamma functions $\Gamma(x+1) / \Gamma(x+s), x>0, s \in(0,1)$ where $\Gamma$ is the gamma function; we refer to $[3,4]$ and the references given therein. One of the most used and most studied inequality about the ratio of gamma function is the Kershaw inequality [5], which can be stated as: For $x>0$ and $0<s<1$

$$
\exp ((1-s) \psi(x+\sqrt{s}))<\frac{\Gamma(x+1)}{\Gamma(x+s)}<\exp \left((1-s) \psi\left(x+\frac{s+1}{2}\right)\right) .
$$

Inspired by this result, Ismail and Muldoon [1] presented the $q$-analogue of the right hand side of (1.1) for the $q$-gamma function: Let $0<q<1$ and $0<s<1$. Then

$$
\frac{\Gamma_{q}(x+1)}{\Gamma_{q}(x+s)}<\exp \left((1-s) \psi_{q}\left(x+\frac{s+1}{2}\right)\right)
$$

hold true for all $x>-s$ where $\Gamma_{q}$ is the $q$-gamma function defined for all positive real $x$ as $[6,7]$

$$
\Gamma_{q}(x)=|1-q|^{1-x} q^{\frac{x(x-1)}{2}} H(q-1) \prod_{n=0}^{\infty} \frac{1-\hat{q}^{n+1}}{1-\hat{q}^{n+x}}, \quad 1 \neq q>0,
$$

(c) The Author(s) 2021. This article is licensed under a Creative Commons Attribution 4.0 International License, which permits use sharing, adaptation, distribution and reproduction in any medium or format, as long as you give appropriate credit to the original author(s) and the source, provide a link to the Creative Commons licence, and indicate if changes were made. The images or other third party material in this article are included in the article's Creative Commons licence, unless indicated otherwise in a credit line to the material. If material is not included in the article's Creative Commons licence and your intended use is not permitted by statutory regulation or exceeds the permitted use, you will need to obtain permission directly from the copyright holder. To view a copy of this licence, visit http://creativecommons.org/licenses/by/4.0/. 
and $\psi_{q}$ is the $q$-digamma function defined as the logarithmic derivative of the $q$-gamma function: That is; for all positive real $x$ and $q \neq 1[8,9]$

$$
\psi_{q}(x)=\frac{d}{d x}\left(\log \Gamma_{q}(x)\right)=\frac{\Gamma_{q}^{\prime}(x)}{\Gamma_{q}(x)} .
$$

In (1.3), $|\cdot|$ is the absolute value, $H(\cdot)$ denotes the Heaviside step function and

$$
\hat{q}= \begin{cases}q & \text { if } 0<q \leq 1 \\ q^{-1} & \text { if } q \geq 1\end{cases}
$$

The close connection between two branches of the $q$-gamma function when $0<q<1$ and $q>1$ is given by

$$
\Gamma_{q}(x)=q^{\frac{(x-1)(x-2)}{2}} \Gamma_{q^{-1}}(x), \quad q>1 .
$$

It is worth mentioning that Gao [10] has given a lower bound of the ratio of $q$-gamma functions

$$
\frac{\Gamma_{q}(x+1)}{\Gamma_{q}(x+s)}>\exp \left(\frac{1-s}{2}\left(\psi_{q}(x+1)+\psi_{q}(x+s)\right)\right)
$$

for all $x>0,0 \leq s<1$ and has shown that the lower bound above and the corresponding one of the case $q \rightarrow 1$ in (1.1) are not comparable in general.

Influenced by (1.1), Alzer [2] posed the following open problem: Let $0<q \neq 1$ and $0<$ $s<1$. Determine the best possible values $a(q, s)$ and $b(q, s)$ such that the inequalities

$$
\exp \left[\left((1-s) \psi_{q}(x+a(q, s))\right]<\frac{\Gamma_{q}(x+1)}{\Gamma_{q}(x+s)}<\exp \left[(1-s) \psi_{q}(x+b(q, s))\right]\right.
$$

hold for all $x>0$.

The main goal of this paper is to determine the best possible values $a(q, s)$ and $b(q, s)$ not only when $0<s<1$ but for all real number $s \neq 1$. Our upper bound refines the upper bound of (1.2) and is generalized it for all $0<q \neq 1$. Also, when letting $q \rightarrow 1$, our result comes bach to Kershaw inequality (1.1) for all $0<s<1$ and give a new inequality for gamma function

$$
\exp \left((1-s) \psi\left(x+\frac{s+1}{2}\right)\right)<\frac{\Gamma(x+1)}{\Gamma(x+s)}<\exp ((1-s) \psi(x+\sqrt{s}))
$$

which holds true for all $s>1$. This result is a new inequality for the classical gamma function.

Recently, many contributions have introduced inequalities for $q$-special functions and $q$-integrals (see [11-16] and the references given therein).

\section{Preliminaries and useful lemmas}

A real-valued function $f$, defined on an interval $I$, is called completely monotonic, if $f$ has derivatives of all orders and satisfies

$$
(-1)^{n} f^{(n)}(x) \geq 0, \quad n \in \mathbb{N}_{0} ; x \in I .
$$


A necessary and sufficient condition that $f(x)$ could be called completely monotonic on $(0, \infty)$ is that $[17]$

$$
f(x)=\int_{0}^{\infty} e^{-x t} d \alpha(t)
$$

where $\alpha(t)$ is nondecreasing and the integral converges for $0<x<\infty$.

Recently, numerous papers were published presenting remarkable inequalities involving the $q$-gamma and the $q$-digamma functions by means of investigating the monotonicity and the complete monotonicity properties for functions associated with them (see [18-36] and the extensive list of references given therein).

Ismail and Muldoon [1] provided the equivalent Stieltjes integral representation for the q-digamma function as

$$
\psi_{q}(x)=-\log (1-q)-\int_{0}^{\infty} \frac{e^{-x t}}{1-e^{-t}} d \gamma_{q}(t), \quad x>0,0<q<1
$$

where $\gamma_{q}(t)$ is a discrete measure with positive masses $-\log q$ at the positive points $-k \log q$, $k \in \mathbb{N}$. For completeness, and economy of later statements, they include the value $q=1$ in the definition of $\gamma_{q}(t)$ :

$$
\gamma_{q}(t)= \begin{cases}-\log q \sum_{k=1}^{\infty} \delta(t+k \log q), & 0<q<1 \\ t, & q=1 .\end{cases}
$$

They used the identities

$$
\frac{q^{x} \log q}{1-q^{x}}=-\int_{0}^{\infty} e^{-x t} d \gamma_{q}(t) \text { and } \log \left(1-q^{x}\right)=-\int_{0}^{\infty} \frac{e^{-x t}}{t} d \gamma_{q}(t),
$$

which follow easily from the definition of $d \gamma_{q}(t)$ for all $x>0$ and $0<q<1$.

\section{Lemma 2.1 Let s be a real number and the function}

$$
g(t ; s)=1-\frac{1}{t} \log \left(\frac{e^{(1-s) t}-1}{t(1-s)}\right), \quad s \neq 1,
$$

be defined for all $t>0$. Then the function $t \mapsto g(t ; s)$ is decreasing on $(0, \infty)$ and satisfying $s<g(t ; s)<(s+1) / 2$ if $s<1$ and $1 \leq g(t ; s) \leq(s+1) / 2$ if $s>1$.

Proof Differentiation gives $t^{2} g^{\prime}(t ; s)=h(t)$ where

$$
h(t)=\log \left(\frac{e^{(1-s) t}-1}{t(1-s)}\right)-\frac{(1-s) t e^{(1-s) t}-e^{(1-s) t}+1}{e^{(1-s) t}-1}, \quad s \neq 1 .
$$

Hence, we have

$$
h^{\prime}(t)=-\frac{e^{2(1-s) t}-e^{(1-s) t}\left[t^{2}(1-s)^{2}+2\right]+1}{t\left(e^{(1-s) t}-1\right)^{2}}, \quad s \neq 1 .
$$


When $s<1$, noting $2^{n} \geq n^{2}$ for all $n \geq 4$, we find that

$$
h^{\prime}(t)=-\frac{1}{t\left(e^{(1-s) t}-1\right)^{2}} \sum_{n=4}^{\infty} \frac{(1-s)^{n} t^{n}}{n !}\left(2^{n}-n(n-1)-2\right)<0 .
$$

When $s>1$, we find that

$$
h^{\prime}(t)=-\frac{e^{2(1-s) t}}{t\left(e^{(1-s) t}-1\right)^{2}} \sum_{n=4}^{\infty} \frac{(s-1)^{n} t^{n}}{n !}\left(2^{n}-n(n-1)-2\right)<0 .
$$

These reveal that $h(t)$ is decreasing on $(0, \infty)$. It is not difficult to see that $\lim _{t \rightarrow 0} h(t)=$ 0 which leads to $g(t ; s)$ is decreasing on $(0, \infty)$. The L'Hospital rule evaluates that $\lim _{t \rightarrow 0} g(t ; s)=(s+1) / 2$ and

$$
\lim _{t \rightarrow \infty} g(t ; s)= \begin{cases}s & \text { if } s<1 \\ 1 & \text { if } s>1\end{cases}
$$

which lead to the desired results.

Let $t=-\log q$ in the lemma above. Then we define $b(q, s)$ for all $0<q<1$ and $s \in \mathbb{R}$ as

$$
b(q, s)=g(-\log q ; s)=s+\frac{1}{\log q} \log \left(-\frac{1-q^{1-s}}{(1-s) \log q}\right), \quad s \neq 1 .
$$

Based upon the former lemma, the function $q \mapsto b(q, s)$ is satisfying the inequality $s<$ $b(q, s)<(s+1) / 2$ if $s<1$ and $1<b(q, s)<(s+1) / 2$ if $s>1$.

Lemma 2.2 Let s be a positive real number and the function

$$
\ell(q, s)=-\frac{(1-s)(1-q)\left(1-q^{s}\right) \log q}{q^{s}\left(1-q^{1-s}\right)}, \quad s \neq 1,
$$

be defined for all $0<q<1$. Then we have

$$
\begin{aligned}
& 2+\ell(q, s)-\sqrt{\ell^{2}(q, s)+4 \ell(q, s)}<2 q^{s}, \quad 0<s<1, \\
& 2+\ell(q, s)-\sqrt{\ell^{2}(q, s)+4 \ell(q, s)}<2 q, \quad s>1, \\
& 2+\ell(q, s)-\sqrt{\ell^{2}(q, s)+4 \ell(q, s)}>\frac{2(s-1) q \log q}{1-q^{1-s}}, \quad s>0 .
\end{aligned}
$$

Proof The inequality (2.8) can be rewritten as

$$
2\left(1-q^{s}\right)+\ell(q, s)<\sqrt{\ell^{2}(q, s)+4 \ell(q, s)} .
$$

Squaring both sides gives $q^{s} \ell(q, s) /\left(1-q^{s}\right)^{2}>1$, which is true by the facts that $\left(1-q^{s}\right) /(1-$ $q)<1$ and $1-q^{1-s}<-(1-s) \log q$ for all $0<s<1$. By same method, (2.9) can be proven.

Similarly, the inequality (2.10) is equivalent

$$
-\frac{(1-s) q \log q}{1-q^{1-s}} \ell(q, s)<\left(1+\frac{(1-s) q \log q}{1-q^{1-s}}\right)^{2} .
$$


It suffices to prove that $w(q ; s)>0$ for all $0<s \neq 1$ where

$$
w(q ; s)=\left(1-q^{1-s}\right)^{2}+2(1-s)\left(1-q^{1-s}\right) q \log q-(1-s)^{2} q^{1-s} \log ^{2} q\left(1-q-q^{s}\right)
$$

which can be read as

$$
w(q ; s)=u(y)+(1-s) q v(y) \log q, \quad y=q^{1-s},
$$

where

$$
\begin{aligned}
& u(y)=(1-y)^{2}-y \log ^{2} y=y^{2} \sum_{n=4}^{\infty} \frac{\log ^{n}(1 / y)}{n !}\left[2^{n}-2-n(n-1)\right]>0, \\
& v(y)=2(1-y)+(1+y) \log y=y \sum_{n=3}^{\infty} \frac{\log ^{n}(1 / y)}{n !}(2-n)<0,
\end{aligned}
$$

for all $0<s<1$ and

$$
\begin{aligned}
& u(y)=(1-y)^{2}-y \log ^{2} y=\sum_{n=4}^{\infty} \frac{\log ^{n}(y)}{n !}\left[2^{n}-2-n(n-1)\right]>0, \\
& v(y)=2(1-y)+(1+y) \log y=\sum_{n=3}^{\infty} \frac{\log ^{n}(y)}{n !}(n-2)>0
\end{aligned}
$$

for all $s>1$. These results end the proof.

Lemma 2.3 Let s be a positive real and the two functions

$$
a_{ \pm}(q, s)=\frac{\log \left(1+\frac{1}{2}\left(\ell(q, s) \pm \sqrt{\ell^{2}(q, s)+4 \ell(q, s)}\right)\right)}{\log q}
$$

be defined for all $0<q<1$ where $\ell(q, s)$ is defined as in (2.7). Then we have

$$
\begin{aligned}
& \lim _{q \rightarrow 1} a_{ \pm}(q, s)=\mp \sqrt{s}, \quad s>0, \\
& \lim _{q \rightarrow 0} a_{ \pm}(q, s)= \begin{cases}\mp s, & 0<s<1, \\
\mp 1, & s>1,\end{cases}
\end{aligned}
$$

and

$$
\begin{array}{ll}
a_{+}(q, s)<0<s<a_{-}(q, s)<s+1-b(q, s), & 0<s<1, \\
a_{+}(q, s)<0<1<a_{-}(q, s)<s+1-b(q, s), & s>1,
\end{array}
$$

where $b(q, s)$ is defined in (2.6).

Proof The L'Hospital rule and long simple calculations give

$$
\lim _{q \rightarrow 1} \ell(q, s)=\lim _{q \rightarrow 1} \ell^{\prime}(q, s)=0 \quad \text { and } \quad \lim _{q \rightarrow 1} \ell^{\prime \prime}(q, s)=2 s,
$$


where the derivative is taken with respect to $q$. Hence, we find that

$$
\begin{aligned}
\lim _{q \rightarrow 1} a_{ \pm}(q, s) & =\lim _{q \rightarrow 1} \frac{q \ell^{\prime}(q, s)\left[\sqrt{\ell^{2}(q, s)+4 \ell(q, s)} \pm(\ell(q, s)+2)\right]}{\sqrt{\ell^{2}(q, s)+4 \ell(q, s)}\left[\ell(q, s)+2 \pm \sqrt{\ell^{2}(q, s)+4 \ell(q, s)}\right]} \\
& = \pm \lim _{q \rightarrow 1} \frac{\ell^{\prime}(q, s)}{\sqrt{\ell^{2}(q, s)+4 \ell(q, s)}} \\
& = \pm \lim _{q \rightarrow 1} \frac{\sqrt{\ell^{2}(q, s)+4 \ell(q, s)}}{2 \ell^{\prime}(q, s)} \ell^{\prime \prime}(q, s) \\
& =s / \lim _{q \rightarrow 1} a_{ \pm}(q, s) .
\end{aligned}
$$

It is clear from $(2.11)$ that $a_{+}(q, s)<0$ and $a_{-}(q, s)>0$ which yields $\lim _{q \rightarrow 1} a_{ \pm}(q, s)=\mp \sqrt{s}$.

In order to prove the second limit, it is not difficult to see that $\lim _{q \rightarrow 0} \ell(q, s)=\infty$ and

$$
\begin{aligned}
\ell^{\prime}(q, s) & =-\ell(q, s)\left(\frac{1}{1-q}+\frac{s q^{s-1}}{1-q^{s}}-\frac{1}{q \log q}+\frac{s}{q}-\frac{(1-s) q^{-s}}{1-q^{1-s}}\right) \\
& =-\ell(q, s)\left(\frac{s+q-s q-q^{1-s}}{q(1-q)\left(1 q^{1-s}\right)}+\frac{s q^{s-1}}{1-q^{s}}-\frac{1}{q \log q}\right),
\end{aligned}
$$

which can be used to evaluate

$$
\begin{aligned}
\lim _{q \rightarrow 0} a_{ \pm}(q, s) & = \pm \lim _{q \rightarrow 0} \frac{q \ell^{\prime}(q, s)}{\sqrt{\ell^{2}(q, s)+4 \ell(q, s)}} \\
& =\mp \lim _{q \rightarrow 0} \frac{\ell(q, s)}{\sqrt{\ell^{2}(q, s)+4 \ell(q, s)}}\left(\frac{q}{1-q}+\frac{s q^{s}}{1-q^{s}}-\frac{1}{\log q}+s-\frac{(1-s) q^{1-s}}{1-q^{1-s}}\right) \\
& = \begin{cases}\mp s, & 0<s<1, \\
\mp 1, & s>1 .\end{cases}
\end{aligned}
$$

The inequality $a_{-}(q, s)>s$ is verified for $0<s<1$ by using $(2.8)$ as

$$
a_{-}(q ; s)-s=\frac{\log \left(\frac{2+\ell(q, s)-\sqrt{\ell^{2}(q, s)+4 \ell(q, s)}}{2 q^{s}}\right)}{\log q}>0, \quad 0<s<1,
$$

and the inequality $a_{-}(q, s)>1$ is verified for $s>1$ by using $(2.9)$ as

$$
a_{-}(q ; s)-1=\frac{\log \left(\frac{2+\ell(q, s)-\sqrt{\ell^{2}(q, s)+4 \ell(q, s)}}{2 q}\right)}{\log q}>0, \quad s>1 .
$$

Also, the inequality $a_{-}(q, s)<s+1-b(q, s)$ is verified for $0<s \neq 1$ by using $(2.10)$ as

$$
a_{-}(q, s)-s-1+b(q, s)=\frac{\log \left(\frac{2+\ell(q, s)-\sqrt{\ell^{2}(q, s)+4 \ell(q, s)}}{2} \frac{1-q^{1-s}}{(s-1) q \log q}\right)}{\log q}<0 .
$$

This ends the proof. 


\section{The main results}

In this section, the monotonicity and complete monotonicity properties are investigated as applied to certain functions involving the $q$-gamma and $q$-digamma functions and exploited to provide the best possible values $a(q, s)$ and $b(q, s)$ in (1.7).

Theorem 3.1 Let $s$ and $a$ be real numbers with $s \neq 1$ and the function

$$
\begin{aligned}
F_{a}(x ; q, s)= & \log \Gamma_{q}(x+1)-\log \Gamma_{q}(x+s)-(1-s) \psi_{q}(x+a) \\
& +(1-s)\left(a-\frac{s+1}{2}\right) H(q-1) \log q
\end{aligned}
$$

be defined for all $x>\max \{-a,-1\}$ and $q>0$. Let $b(q, s)$ be defined in (2.6).

- When $s<1$. Then:

1. The function $-F_{a}(x ; q, s)$ is a completely monotonic function on $(-a, \infty)$ if and only if $a \geq b(\hat{q}, s)$.

2. The function $F_{a}(x ; q, s)$ is a completely monotonic function on $(-s, \infty)$ if and only if $a \leq s$.

- When $s>1$. Then:

1. The function $-F_{a}(x ; q, s)$ is a completely monotonic function on $(-1, \infty)$ if and only if $a \leq 1$.

2. The function $F_{a}(x ; q, s)$ is a completely monotonic function on $(-1, \infty)$ if and only if $a \geq b(\hat{q}, s)$.

Proof Differentiation gives

$$
F_{a}^{\prime}(x ; q, s)=\psi_{q}(x+1)-\psi_{q}(x+s)-(1-s) \psi_{q}^{\prime}(x+a)
$$

which can be represented, by using (2.3), as

$$
F_{a}^{\prime}(x ; q, s)=-\int_{0}^{\infty} \frac{e^{-(x+1) t}}{1-e^{-t}} f_{s}(a, t) d \gamma_{q}(t), \quad 0<q<1 .
$$

Hence, for a positive integer $n$, we have

$$
(-1)^{n} F_{a}^{(n+1)}(x ; q, s)=-\int_{0}^{\infty} \frac{t^{n} e^{-(x+1) t}}{1-e^{-t}} f_{s}(a, t) d \gamma_{q}(t),
$$

where

$$
f_{s}(a, t)=1-e^{(1-s) t}+(1-s) t e^{(1-a) t}
$$

A partial derivative with respect to $a$ gives

$$
\frac{\partial}{\partial a} f_{s}(a, t)=-(1-s) t^{2} e^{(1-a) t}
$$

which means that the function $a \mapsto f_{s}(a, t)$ is decreasing on $\mathbb{R}$ if $s<1$ and increasing on $\mathbb{R}$ if $s>1$ for all $t>0$. It is easy to show that $f_{s}(s, t)>0$ and $f_{s}(1, t)<0$ for all real $s \neq 1$ and $t>0$, which means that there is a unique root lying between $s$ and 1 at $a=g(t ; s)$ where $g(t ; s)$ is defined in (2.5), such that: 
In the case of $s<1$ : The function $f_{s}(a, t)>0$ for all $a<g(t ; s)$ and $f_{s}(a, t)<0$ for all $a>$ $g(t ; s)$. According to the definition of the discrete measure $f_{s}(a, t) d \gamma_{q}$, the function $F_{a}^{\prime}(x ; q, s)$ is a completely monotonic function on $(-a, \infty)$ if $f_{s}(a, t)<0$ at $t=-k \log q$, $k \in \mathbb{N}$ and $-F_{a}^{\prime}(x ; q, s)$ is a completely monotonic function on $(-a, \infty)$ if $f_{s}(a, t)>0$ at $t=-k \log q, k \in \mathbb{N}$. In other words, $F_{a}^{\prime}(x ; q, s)$ is a completely monotonic function on $(-a, \infty)$ if $a \geq g(-k \log q ; s)$ and $-F_{a}^{\prime}(x ; q, s)$ is a completely monotonic function on $(-a, \infty)$ if $a \leq g(-k \log q ; s)$. By virtue of Lemma 2.1, the function $t \mapsto g(t ; s)$ is decreasing on $(0, \infty)$, then $g(-k \log q ; s)$ is decreasing for all $k \in \mathbb{N}$, which means that the function $F_{a}^{\prime}(x ; q, s)$ is a completely monotonic function on $(-a, \infty)$ if $a \geq b(q, s)$ and $-F_{a}^{\prime}(x ; q, s)$ is a completely monotonic function on $(-s, \infty)$ if $a \leq s$.

In the case of $s>1$ : The function $f_{s}(a, t)<0$ for all $a<g(t ; s)$ and $f_{s}(a, t)>0$ for all $a>$ $g(t ; s)$. Thus, the function $F_{a}^{\prime}(x ; q, s)$ is a completely monotonic function on $(-1, \infty)$ if $a \leq 1$ and $-F_{a}^{\prime}(x ; q, s)$ is a completely monotonic function on $(-1, \infty)$ if $a \geq b(q, s)$.

It is not difficult to see from (1.4) and (1.5) that $F_{a}^{\prime}\left(x ; q^{-1}, s\right)=F_{a}^{\prime}(x ; q, s)$ for all $q>1$ and so the former rules can be extended to $0<q \neq 1$.

By (1.3) and (1.4), it is easy to rewrite the function in (3.1) in the form

$$
F_{a}(x ; q, s)=\sum_{n=0}^{\infty} \log \left[\frac{1-\hat{q}^{n+x+s}}{1-\hat{q}^{n+x+1}}\right]-(1-s) \sum_{n=0}^{\infty} \frac{\hat{q}^{n+x+a} \log \hat{q}}{1-\hat{q}^{n+x+a}}, \quad q \neq 1 .
$$

Hence, $\lim _{x \rightarrow \infty} F_{a}(x ; q, s)=0$ for all real $s \neq 1$ which means that for all positive real $q \neq 1$ :

- When $s<1$, the function $-F_{a}(x ; q, s)$ is a completely monotonic function on $(-a, \infty)$ if $a \geq b(\hat{q}, s)$ and $F_{a}(x ; q, s)$ is a completely monotonic function on $(-s, \infty)$ if $a \leq s$.

- When $s>1$ the function $-F_{a}(x ; q, s)$ is a completely monotonic function on $(-1, \infty)$ if $a \leq 1$ and $F_{a}(x ; q, s)$ is a completely monotonic function on $(-1, \infty)$ if $a \geq b(\hat{q}, s)$.

Conversely, in order to prove the converse, it is clear from (3.2) that

$$
\lim _{x \rightarrow \infty} \hat{q}^{-x} F_{a}(x ; q, s)=-\frac{\hat{q}^{s}\left(1-\hat{q}^{1-s}\right)+(1-s) \hat{q}^{a} \log \hat{q}}{1-\hat{q}}, \quad q \neq 1 .
$$

When $s<1$, let the function $-F_{a}(x ; q, s)$ be a completely monotonic function on $(-a, \infty)$. Thus $\lim _{x \rightarrow \infty} \hat{q}^{-x} F_{a}(x ; q, s) \leq 0$, which yields $a \geq b(\hat{q}, s)$. Now, suppose $F_{a}(x ; q, s)$ (with $a>$ $s$ ) is a completely monotonic function on $(-s, \infty)$ for all positive $q \neq 1$. This means that $F_{a}(x ; q, s)$ is positive on $(-s, \infty)$. But this contradicts $\lim _{x \rightarrow-s}=-\infty, a>s$.

When $s>1$, the proof is similar. This ends the proof.

Corollary 3.1 Let a real number $s \neq 1$ and a positive real number $q \neq 1$. Then, when $s<1$, the inequalities

$$
\begin{aligned}
q^{(s-1)\left(a-\frac{s+1}{2}\right) H(q-1)} \exp \left((1-s) \psi_{q}(x+a)\right) & <\frac{\Gamma_{q}(x+1)}{\Gamma_{q}(x+s)} \\
& <q^{(s-1)\left(b-\frac{s+1}{2}\right) H(q-1)} \exp \left((1-s) \psi_{q}(x+b)\right)
\end{aligned}
$$

hold true for all $x>-s, a \leq s$ and $b \geq b(\hat{q}, s)$ with best possible constants $a=s$ and $b=b(\hat{q}, s)$. When $s>1$, the inequalities (3.5) are reversed for all $x>-1, a \leq 1$ and $b \geq b(\hat{q}, s)$ with best possible constants $a=1$ and $b=b(\hat{q}, s)$. 
Proof The results obtained in Theorem 3.1 refer to $F_{b}(x ; q, s)<0<F_{a}(x ; q, s)$ for all $x>-s$, $a \leq s$ and $b \geq b(\hat{q}, s)$ when $s<1$, which is equivalent to (3.5) and $F_{a}(x ; q, s)<0<F_{b}(x ; q, s)$ for all $x>-1, a \leq 1$ and $b \geq b(\hat{q}, s)$ when $s>1$, which is equivalent to the reverse.

Corollary 3.2 Let a real number $s \neq 1$, a positive real number $q \neq 1$ and a non-negative integer $n$. Then, when $s<1$, the inequalities

$$
\begin{aligned}
(-1)^{n}(1-s) \psi_{q}^{(n+1)}(x+b) & <(-1)^{n}\left[\psi_{q}^{(n)}(x+1)-\psi_{q}^{(n)}(x+s)\right] \\
& <(-1)^{n}(1-s) \psi_{q}^{(n+1)}(x+a)
\end{aligned}
$$

hold true for all $x>-s, a \leq s$ and $b \geq b(\hat{q}, s)$ with best possible constants $a=s$ and $b=b(\hat{q}, s)$. When $s>1$, the inequalities (3.6) are reversed for all $x>-1, a \leq 1$ and $b \geq b(\hat{q}, s)$ with best possible constants $a=1$ and $b=b(\hat{q}, s)$.

Proof The results obtained in Theorem 3.1 refer to

$$
(-1)^{n} F_{a}^{(n+1)}(x ; q, s)<0<(-1)^{n} F_{b}^{(n+1)}(x ; q, s)
$$

for all $x>-s, a \leq s$ and $b \geq b(\hat{q}, s)$ when $s<1$, which is equivalent to (3.6) and $F_{b}^{\prime}(x ; q, s)<$ $0<F_{a}(x ; q, s)$ for all $x>-1, a \leq 1$ and $b \geq b(\hat{q}, s)$ when $s>1$, which is equivalent to the reverse.

Remark 3.1 When $0<q, s<1$, Ismail and Muldoon [1] established the upper bound as in (1.2). By virtue of Lemma $2.1, b(q, s)<(s+1) / 2$ and by increasing of the $q$-digamma and exponential functions, it turns out that the upper bound of (3.5) is less (better) than of the upper bound of (1.2).

Remark 3.2 When letting $q \rightarrow 1$ and $0<s<1$, the inequalities (3.5) become

$$
\exp ((1-s) \psi(x+s))<\frac{\Gamma(x+1)}{\Gamma(x+s)}<\exp \left((1-s) \psi\left(x+\frac{s+1}{2}\right)\right)
$$

which are true for all $x>-s$. It is noted that the upper bound is the same as (1.1) but the lower bound of (1.1) is bigger (better) than the lower bound of (3.7). Motivated by this, we devote the following theorem to refining the lower bound of (3.5) to a $q$-extension of the lower bound of (1.1).

Theorem 3.2 Let $s \neq 1$ and $q \neq 1$ be positive real numbers, $b(q, s)$ be defined in (2.6) and $a_{ \pm}(q, s)$ be defined in (2.11). Then:

- When $0<s<1$, the inequalities (3.5) hold true for all $x \geq 0, a_{+}(\hat{q}, s) \leq a \leq a_{-}(\hat{q}, s)$ and $b(\hat{q}, s) \leq b \leq 1+s-b(\hat{q}, s)$ with best possible constants $a=a_{-}(\hat{q}, s)$ and $b=b(\hat{q}, s)$.

- When $s>1$, the inequalities (3.5) are reversed for all $x \geq 0, a_{+}(\hat{q}, s) \leq a \leq a_{-}(\hat{q}, s)$ and $b(\hat{q}, s) \leq b \leq 1+s-b(\hat{q}, s)$ with best possible constants $a=a_{-}(\hat{q}, s)$ and $b=b(\hat{q}, s)$.

Proof Let $0<s<1$ and the function

$$
f_{a}(x ; q, s)=F_{a}(x ; q, s)-F_{a}(x+1 ; q, s)=-\log [x+1]_{q}+\log [x+s]_{q}-(1-s) \frac{q^{x+a} \log q}{1-q^{x+a}},
$$


where $F_{a}(x ; q, s)$ is defined in (3.1). Then we have

$$
f_{a}^{\prime}(x ; q, s)=-\frac{q^{x} \log q}{\left(1-q^{x+1}\right)\left(1-q^{x+s}\right)\left(1-q^{x+a}\right)^{2}} f(x),
$$

where

$$
f(x)=q^{s}\left(1-q^{1-s}\right)\left(1-q^{x+a}\right)^{2}+(1-s) q^{a}\left(1-q^{x+1}\right)\left(1-q^{x+s}\right) \log q
$$

which can be rewritten as

$$
f(x)=q^{a+s}\left[\lambda_{a}(q, s)+\mu_{a}(q, s) q^{2 x}-v(q, s) q^{x}\right]
$$

where

$$
\begin{aligned}
& \lambda_{a}(q, s)=q^{-a}\left(1-q^{1-s}\right)+(1-s) q^{-s} \log q, \\
& \mu_{a}(q, s)=q^{a}\left(1-q^{1-s}\right)+(1-s) q \log q, \\
& v(q, s)=2\left(1-q^{1-s}\right)+(1-s)\left(1+q^{1-s}\right) \log q .
\end{aligned}
$$

It is easy to see that

$$
v(q, s)=q^{1-s} \sum_{n=3}^{\infty} \frac{(1-s)^{n} \log ^{n}(1 / q)}{n !}(2-n)<0, \quad 0<q<1
$$

which means that $f(x)>0$ for all $0<q<1$ if $\lambda_{a}(q, s) \geq 0$ and $\mu_{a}(q, s) \geq 0$. In other words: $f(x)>0$ for all $0<q<1$ if

$$
b(q, s) \leq a \leq s+1-b(q, s)
$$

which shows that $f_{a}(x ; q, s)$ is increasing on $(-s, \infty)$ and since $\lim _{x \rightarrow \infty} f_{a}(x ; q, s)=0$, we have $f_{a}(x ; q, s)<0$ for all $0<q<1$ and $a$ satisfies (3.9). Hence, we have

$$
F_{a}(x ; q, s)<F_{a}(x+1 ; q, s)<\lim _{n \rightarrow \infty} F_{a}(x+n ; q, s)=0,
$$

which gives the upper bound of (3.5) for all positive real $q \neq 1$ since $F_{a}(x ; q, s)=F_{a}\left(x ; q^{-1}, s\right)$. Based on Lemma 2.1, $b(q, s) \leq(s+1) / 2$, then $a=b(\hat{q}, s)$ is better than $a=s+1-b(\hat{q}, s)$.

In order to prove the lower bound, differentiating (3.8) gives

$$
f^{\prime}(x)=q^{x+a+s} \log q\left[2 \mu_{a}(q, s) q^{x}-v(q, s)\right]<0, \quad \mu_{a}(q, s) \geq 0,
$$

which leads to $f(x)$ is decreasing on $[0, \infty)$ for all $0<q<1$ and $a \leq s+1-b(q, s)$. Hence, $f(x)<0$ if $f(0) \leq 0$. That is,

$$
q^{2 a}-(\ell(q, s)+2) q^{a}+1 \leq 0
$$


which yields $a_{+}(q, s) \leq a \leq a_{-}(q, s)$. By virtue of $(2.14) a_{-}(q, s)<s+1-b(q, s)$, thus we have to take $a_{+}(q, s) \leq a \leq a_{-}(q, s)$. By increasing of the $q$-digamma and exponential functions, the best value is at $a=a_{-}(q, s)$ and this result can be extended for all $q>0$ at $a=a_{-}(\hat{q}, s)$.

When $s>1$, by the same method and by aiding (2.15), we can easily prove the reverse of (3.5).

Remark 3.3 It is clear that Theorem 3.2 and Corollary 3.1 have the same upper bounds when $0<s<1$ and the same lower bounds when $s>1$. By virtue of Lemma $2.3\left(a_{-}(\hat{q}, s)<s\right.$ when $0<s<1$ and $a_{-}(\hat{q}, s)<1$ when $\left.s>1\right)$, then the results in Theorem 3.2 are better than the corresponding ones in Corollary 3.1.

Remark 3.4 It is worth mentioning that when letting $q \rightarrow 1$ :

- When $0<s<1$, the results in Theorem 3.2 go back to the Kershaw inequalities (1.1) by using the limits in Lemmas 2.1 and 2.2.

- When $s>1$, the results in Theorem 3.2 take the form of the inequality (1.8), which is considered as the extension of the Kershaw inequality (1.1) when $s>1$. This result is a new inequality for the ratio of classical gamma functions.

Acknowledgements

The authors would like to thank the anonymous referees for their valuable comments and suggestions, which helped to improve the paper.

\section{Funding}

This project was funded by the Deanship of Scientific Research (DSR), King Abdulaziz University, Jeddah, under grant No. (KEP-8-130-38). The authors, therefore, acknowledge with thanks DSR technical and financial support.

Availability of data and materials

Not applicable.

Competing interests

The authors declare that they have no competing interests.

Authors' contributions

All authors contributed equally and significantly in writing this paper. All authors have read and agreed to the final version of the manuscript. All authors read and approved the final manuscript.

\section{Author details}

'Department of Mathematics, Faculty of Science, King Abdulaziz University, P.O. Box 80203, Jeddah, 21589, Saudi Arabia.

${ }^{2}$ Unaizah Faculty of Arts and Sciences, Qassim University, P.O. Box 3771, Unaizah, Qassim 51431, Saudi Arabia.

\section{Publisher's Note}

Springer Nature remains neutral with regard to jurisdictional claims in published maps and institutional affiliations.

Received: 31 May 2019 Accepted: 6 June 2021 Published online: 12 June 2021

\section{References}

1. Ismail, M.E.H., Muldoon, M.E.: Inequalities and monotonicity properties for gamma and q-gamma functions, approximation and computation. In: Internat. Ser. Numer. Math., vol. 119, pp. 309-323. Birkhäuser, Boston (1994)

2. Alzer, H.: Sharp bounds for the ratio of q-gamma functions. Math. Nachr. 222(1), 5-14 (2001)

3. Mortici, C.: New approximation formulas for evaluating the ratio of gamma functions. Math. Comput. Model. 52, 425-433 (2010)

4. Alzer, H.: On some inequalities for the gamma and psi functions. Math. Comput. 66, 373-389 (1997)

5. Kershaw, D.: Some extensions of $W$ Gautschi's inequalities for the gamma function. Math. Comput. 41, 607-611 (1983)

6. Moak, D.S.: The $q$-gamma function for $q>1$. Aequ. Math. 20, 278-285 (1980)

7. Askey, R.: The q-gamma and $q$-beta functions. Appl. Anal. 8, 125-141 (1978)

8. Krattenthaler, C., Srivastava, H.M.: Summations for basic hypergeometric series involving a $q$-analogue of the digamma function. Comput. Math. Appl. 32(2), 73-91 (1996)

9. Salem, A.: Some properties and expansions associated with q-digamma function. Quaest. Math. 36(1), 67-77 (2013)

10. Gao, P.: Some monotonicity properties of the $q$-gamma function. RGMIA Res. Rep. Collect., 8(3), Article ID 4 (2005) 
11. Baleanu, D., Agarwal, P.: Certain inequalities involving the fractional $q$-integral operators. Abstr. Appl. Anal. 2014 Article ID 371274, 10 pages (2014). https://doi.org/10.1155/2014/371274

12. Sitho, S., Ntouyas, S.K., Agarwal, P., Tariboon, J.: Noninstantaneous impulsive inequalities via conformable fractional calculus. J. Inequal. Appl. 2018, 261 (2018). https://doi.org/10.1186/s13660-018-1855-z

13. Abdeldaim, A., El-Deeb, A.A., Agarwal, P., El-Sennary, H.A.: On some dynamic inequalities of Steffensen type on time scales. Math. Methods Appl. Sci. 41(12), 4737-4753 (2018). https://doi.org/10.1002/mma.4927

14. Choi, J., Agarwal, P.: Certain fractional integral inequalities involving hypergeometric operators. East Asian Math. J. 30(3), 283-291 (2014). https://doi.org/10.7858/eamj.2014.018

15. Agarwal, P., Choi, J.: Certain fractional integral inequalities associated with pathway fractional integral operators. Bull. Korean Math. Soc. 53(1), 181-193 (2016). https://doi.org/10.4134/BKMS.2016.53.1.181

16. Salem, A.: Best bounds for the Lambert W functions. J. Math. Inequal. 14(4), 1237-1247 (2020). https://doi.org/10.7153/jmi-2020-14-80

17. Widder, D.V:: The Laplace Transform. Princeton University Press, Princeton (1941)

18. Batir, N.: Monotonicity properties of $q$-digamma and $q$-trigamma functions. J. Approx. Theory 192, 336-346 (2015)

19. Batir, N.: q-Extensions of some estimates associated with the digamma function. J. Approx. Theory 174, 54-64 (2013)

20. Qi, F:: Complete monotonicity of functions involving the $q$-trigamma and $q$-tetragamma functions. Rev. R. Acad. Cienc. Exactas Fís. Nat., Ser. A Mat. 109(2), 419-429 (2015)

21. Salem, A.: Sharp lower and upper bounds for the 9 -gamma function. Math. Inequal. Appl. 23(3), 855-872 (2020)

22. Salem, A.: Sharp bounds for the $q$-gamma function in terms of the Lambert $W$ function. Ramanujan J. 49, 321-339 (2019)

23. Salem, A., Alzahrani, F.: Improvements of bounds for the $q$-gamma and the $q$-polygamma functions. J. Math. Inequal. 11(3), 873-883 (2017)

24. Salem, A.: Completely monotonic functions related to the gamma and the $q$-gamma functions. Rev. R. Acad. Cienc. Exactas Fís. Nat. Ser. A Mat. 111(1), 271-280 (2017)

25. Salem, A.: Some classes of completely monotonic functions related to $q$-gamma and $q$-digamma functions. Math Inequal. Appl. 19(3), 853-862 (2016)

26. Salem, A.: A certain class of approximations for the q-digamma function. Rocky Mt. J. Math. 46(5), 1665-1677 (2016)

27. Salem, A:: Monotonic functions related to the 9 -gamma function. Monatshefte Math. 179(2), 281-292 (2016)

28. Salem, A.: Some completely monotonic functions associated with the q-gamma and the $q$-polygamma functions. Acta Math. Sci. 35(5), 1214-1224 (2015)

29. Salem, A.: Completely monotonic functions related to the $q$-gamma and the $q$-trigamma functions. Anal. Appl. 13(2) 125-134 (2015)

30. Salem, A.: Complete monotonicity properties of functions involving $q$-gamma and $q$-digamma functions. Math Inequal. Appl. 17(3), 801-811 (2014)

31. Salem, A.: Two classes of bounds for the $q$-gamma and the $q$-digamma functions in terms of the $q$-zeta functions. Banach J. Math. Anal. 8(1), 109-117 (2014)

32. Salem, A.: An infinite class of completely monotonic functions involving the q-gamma function. J. Math. Anal. Appl. 406(2), 392-399 (2013)

33. Salem, A.: A completely monotonic function involving $q$-gamma and $q$-digamma functions. J. Approx. Theory 164(7), 971-980 (2012)

34. Grinshpan, A.Z., Ismail, M.E.H.: Completely monotonic functions involving the gamma and q-gamma functions. Proc. Am. Math. Soc. 134, 1153-1160 (2006)

35. Elezovic, N., Giordano, C., Pecaric, J.: Convexity and q-gamma function. Rend. Circ. Mat. Palermo XLVIII, 285-298 (1999)

36. Ismail, M.E.H., Lorch, L., Muldoon, M.E.: Completely monotonic functions associated with the gamma function and its q-analogues. J. Math. Anal. Appl. 116, 1-9 (1986)

\section{Submit your manuscript to a SpringerOpen ${ }^{\circ}$ journal and benefit from:}

- Convenient online submission

- Rigorous peer review

- Open access: articles freely available online

- High visibility within the field

- Retaining the copyright to your article

Submit your next manuscript at $\gg$ springeropen.com 